TRANSACTIONS OF THE

AMERICAN MATHEMATICAL SOCIETY

Volume 362, Number 6, June 2010, Pages 3161-3182

S 0002-9947(10)04856-7

Article electronically published on January 20, 2010

\title{
SPECTRAL AND DYNAMICAL PROPERTIES OF CERTAIN RANDOM JACOBI MATRICES WITH GROWING PARAMETERS
}

\author{
JONATHAN BREUER
}

\begin{abstract}
In this paper, a family of random Jacobi matrices with off-diagonal terms that exhibit power-law growth is studied. Since the growth of the randomness is slower than that of these terms, it is possible to use methods applied in the study of Schrödinger operators with random decaying potentials. A particular result of the analysis is the existence of operators with arbitrarily fast transport whose spectral measure is zero dimensional. The results are applied to the infinite Dumitriu-Edelman model (2002), and its spectral properties are analyzed.
\end{abstract}

\section{INTRODUCTION}

For a sequence of positive numbers, $\{a(n)\}_{n=1}^{\infty}$, and a sequence, $\{b(n)\}_{n=1}^{\infty}$, of real numbers, let $J\left(\{a(n)\}_{n=1}^{\infty},\{b(n)\}_{n=1}^{\infty}\right)$ denote the Jacobi matrix with off-diagonal elements given by $\{a(n)\}_{n=1}^{\infty}$ and diagonal elements given by $\{b(n)\}_{n=1}^{\infty}$ on the diagonal. That is,

$$
J\left(\{a(n)\}_{n=1}^{\infty},\{b(n)\}_{n=1}^{\infty}\right)=\left(\begin{array}{ccccc}
b(1) & a(1) & 0 & 0 & \cdots \\
a(1) & b(2) & a(2) & 0 & \cdots \\
0 & a(2) & b(3) & a(3) & \ddots \\
\vdots & \ddots & \ddots & \ddots & \ddots
\end{array}\right) .
$$

For $\eta_{1} \in(0,1)$ and $\lambda_{1}>0$, let $J_{\lambda_{1}, \eta_{1}}$ be the Jacobi matrix whose parameters are $a_{\lambda_{1}, \eta_{1}}(n)=\lambda_{1} n^{\eta_{1}}$ and $b_{\lambda_{1}, \eta_{1}}(n) \equiv 0$. Since $\eta_{1}<1, J_{\eta_{1}}$ is a self-adjoint operator on $\ell^{2}(\mathbb{N})[1]$. For any such operator we may define the spectral measure, $\mu$, as the unique measure satisfying

$$
\left(\delta_{1},(J-z)^{-1} \delta_{1}\right)=\int_{\mathbb{R}} \frac{d \mu(x)}{x-z} \quad z \in \mathbb{C} \backslash \mathbb{R},
$$

where $(\cdot, \cdot)$ denotes the inner product in $\ell^{2}$. It follows from the work of Janas and Naboko [8] that $J_{\lambda_{1}, \eta_{1}}$ has absolutely continuous spectrum covering the whole real line.

This paper deals with random perturbations of $J_{\lambda_{1}, \eta_{1}}$ that are weak in the sense that the variance of the perturbing random parameters grows like $\sim n^{\eta_{2}}$ with $\eta_{2}<\eta_{1}$.

Received by the editors November 13, 2007 and, in revised form, June 16, 2008.

2000 Mathematics Subject Classification. Primary 47B36; Secondary 60H25.

(C)2010 American Mathematical Society Reverts to public domain 28 years from publication 
The case $a(n) \equiv 1, \eta_{2}<0$ (" $\eta_{1}=0$ ") and no perturbation off the diagonal is the extensively studied family of one-dimensional discrete Schrödinger operators with a random decaying potential [3, 4, 5, 13, 14, 19. For these Schrödinger operators it has been established that when $\eta_{2}<-\frac{1}{2}$, the absolutely continuous spectrum of the Laplacian is a.s. preserved. When $\eta_{2}>-\frac{1}{2}$, however, the disorder wins over, and the spectrum is pure point with eigenfunctions that decay at a super-polynomial rate. At the critical point $\left(\eta_{2}=-\frac{1}{2}\right)$ the spectral behavior exhibits a sensitive dependence on the coupling constant: The generalized eigenfunctions decay polynomially, and the spectral measure is pure point or singular continuous according to whether these generalized eigenfunctions are in $\ell^{2}$ or not (for a comprehensive treatment of discrete Schrödinger operators with random decaying potentials, see Section 8 of [13]).

From this perspective, the extension presented in this paper is in allowing growth of the off-diagonal terms. Intuitively, these terms are responsible for transport, and thus, their growth should have an effect on the spectrum similar to that of the decay of the potential (diagonal terms). Indeed, a particular case of our analysis is that of $\eta_{2}=0$, namely, the diagonal terms are i.i.d. random variables. We show that the critical point here is $\eta_{1}=\frac{1}{2}$. Below this value, the spectrum is a.s. pure point, whereas above it the spectral measure is one-dimensional.

More generally, let $\left\{X_{\omega}(n)\right\}_{n=1}^{\infty}$ be a sequence of i.i.d. random variables. Let $\left\{Y_{\omega}(n)\right\}_{n=1}^{\infty}$ be another such sequence (the distributions of the $X$ 's and the $Y$ 's need not be the same). Assume the following is satisfied:

(i) For all $n$

$$
\left\langle X_{\omega}(n)\right\rangle=\left\langle Y_{\omega}(n)\right\rangle=0
$$

(where $\langle f(\omega)\rangle \equiv \int_{\Omega} f(\omega) d p$ and $(\Omega, \mathcal{F}, d p)$ is the underlying probability space).

(ii) For any $k \in \mathbb{N}$

$$
\left\langle\left|Y_{\omega}(n)\right|^{k}\right\rangle<\infty, \quad\left\langle\left|X_{\omega}(n)\right|^{k}\right\rangle<\infty .
$$

$$
\left\langle\left(X_{\omega}(n)\right)^{2}\right\rangle=1, \quad\left\langle\left(Y_{\omega}(n)\right)^{2}\right\rangle=\frac{1}{4} .
$$

(iv) The common distribution of $X_{\omega}(n)$ is absolutely continuous with respect to Lebesgue measure.

Given the quadruple $\Upsilon=\left(\eta_{1}, \eta_{2}, \lambda_{1}, \lambda_{2}\right)$ with $0<\eta_{1}<1, \eta_{2}<\eta_{1}$ and $\lambda_{1}, \lambda_{2}>0$, define the two random sequences

$$
b_{\Upsilon, \omega}(n) \equiv b_{\lambda_{2}, \eta_{2} ; \omega}(n) \equiv \lambda_{2} n^{\eta_{2}} X_{\omega}(n), \quad \alpha_{\lambda_{2}, \eta_{2} ; \omega}(n) \equiv \lambda_{2} n^{\eta_{2}} Y_{\omega}(n) .
$$

Let

$$
a_{\Upsilon, \omega}(n) \equiv a_{\lambda_{1}, \eta_{1}}(n)+\alpha_{\lambda_{2}, \eta_{2} ; \omega}(n)
$$

and define

$$
J_{\Upsilon, \omega}=J\left(\left\{a_{\Upsilon, \omega}(n)\right\}_{n=1}^{\infty},\left\{b_{\Upsilon, \omega}(n)\right\}_{n=1}^{\infty}\right) .
$$

The assumptions on the parameters defining $J_{\Upsilon, \omega}$ do not exclude the possibility that some of the off-diagonal terms will vanish. However, with probability one, this 
may happen only a finite number of times, so that $J_{\Upsilon, \omega}$ has an infinite part with strictly positive off-diagonal entries. In the following, when we refer to $J_{\Upsilon, \omega}$, we refer to this part.

We shall prove

Theorem 1.1. For the model above, let $\gamma=\eta_{1}-\eta_{2}$ and let $\Lambda=\frac{1}{2}\left(\lambda_{2} / \lambda_{1}\right)^{2}$. The following holds with probability one:

(1) If $\gamma>\frac{1}{2}$ the spectrum of $J_{\Upsilon, \omega}$ is $\mathbb{R}$ and $\mu_{\Upsilon, \omega}$, the spectral measure of $J_{\Upsilon, \omega}$, is one-dimensional, meaning that it does not give weight to sets of Hausdorff dimension less than 1.

(2) In the case $\gamma=\frac{1}{2}$ the spectrum of $J_{\Upsilon, \omega}$ is $\mathbb{R}$, and we have the following two possibilities:

(a) If $\Lambda>1-\eta_{1}$, then $\mu_{\Upsilon, \omega}$ is pure point with eigenfunctions decaying like

$$
\left|\psi_{\omega}^{E}(n)\right|^{2} \sim n^{-\left(\Lambda+\eta_{1}\right)}
$$

(b) If $\Lambda \leq 1-\eta_{1}$, then $\mu_{\Upsilon, \omega}$ is purely singular continuous with exact Hausdorff dimension equal to $1-\frac{\Lambda}{\left(1-\eta_{1}\right)}$.

(3) If $\gamma<\frac{1}{2}$, then the spectrum is pure point with eigenfunctions decaying like

$$
\left|\psi_{\omega}^{E}(n)\right|^{2} \sim e^{-\Lambda n^{1-2 \gamma}}
$$

In this case, if $\eta_{1}>2 \eta_{2}$, then the spectrum fills $\mathbb{R}$.

Remark. We say that a measure, $\mu$, has exact Hausdorff dimension, $\varrho$, if it is supported on a set of Hausdorff dimension $\varrho$ and does not give weight to sets of Hausdorff dimension less than $\varrho$. For more information concerning the decomposition of general measures with respect to their Hausdorff-dimensional properties, consult [15] and the references therein.

Remark. Similar to the Schrödinger case, one would expect to have an absolutely continuous spectrum for $\gamma>1 / 2$. Unfortunately, though we believe this is true, one-dimensional spectral measure is all we could get.

Remark. The requirement that $\left\{X_{\omega}(n)\right\}_{n=1}^{\infty}$ and $\left\{Y_{\omega}(n)\right\}_{n=1}^{\infty}$ be identically distributed sequences is not really necessary and is noted here only to simplify the discussion.

In resemblance of the Schrödinger case, the proof of this theorem follows by analyzing the asymptotics of solutions to the formal eigenfunction equation " $J \psi=$ $E \psi$ ". Namely, we shall analyze the solutions to the difference equation

$$
a(n) \psi(n+1)+b(n) \psi(n)+a(n-1) \psi(n-1)=E \psi(n), \quad n>1 .
$$

By a theorem of Kiselev and Last [12, Theorem 1.2], the results obtained have implications for the quantum dynamics associated with $J$; namely the behavior of a given vector $\psi$ under the operation of the one parameter unitary group $e^{-i t J}$. More precisely, let $\hat{X}$ be the position operator defined by

$$
(\hat{X} \psi)(n)=n \psi(n) .
$$


Theorem 1.2 of [12, which can be seen to hold in our setting, says that Theorem 1.1 and Proposition 2.7 below lead to

Theorem 1.2. Assume $\gamma=\frac{1}{2}$ and $\Lambda \leq 1-\eta_{1}$ (namely, case 2(b) of Theorem 1.1). Then for any $\varepsilon>0, m \in \mathbb{N}, T>0$ and $\psi \in \ell^{2}$,

$$
\frac{1}{T} \int_{0}^{T}\left|\left(\hat{X}^{m} e^{-i t J_{\Upsilon, \omega}} \psi, e^{-i t J_{\Upsilon, \omega}} \psi\right)\right| d t \geq C(\omega, \psi, m, \varepsilon) T^{\left(m /\left(1-\eta_{1}\right)\right)-\varepsilon}
$$

with probability one.

Note that $\eta_{1}$ may be chosen arbitrarily close to 1 , while the spectral measure may have any dimension in $[0,1)$. Thus, by tuning the parameters we obtain operators with any local spectral dimensions having arbitrarily fast transport.

As an application of our general analysis, we study the Gaussian $\beta$ ensembles arising naturally in the context of Random Matrix Theory: The eigenvalue distribution functions for the three classical Gaussian ensembles are given by

$$
f_{\beta, N}\left(E_{1}, \cdots, E_{N}\right)=\frac{1}{G_{\beta N}} \exp \left(-\frac{1}{2} \sum_{j=1}^{N} E_{j}^{2}\right) \prod_{1 \leq j<k \leq N}\left|E_{j}-E_{k}\right|^{\beta}
$$

with $\beta=1,2$ and 4 for the Gaussian Orthogonal Ensemble, Gaussian Unitary Ensemble and Gaussian Symplectic Ensemble, respectively.

A family of random matrix ensembles, indexed by $\beta$, having $f_{\beta, N}$ as their eigenvalue distribution function, and for any positive value of $\beta$, was recently constructed by Dumitriu and Edelman [6]. The matrices in these ensembles are finite random Jacobi matrices with the distribution of the off diagonal terms depending on $\beta$ :

Definition 1.3. Fix $\beta>0$. The random family of Jacobi matrices $J_{\beta, \omega} \equiv$ $J\left(\left\{a_{\beta, \omega}(n)\right\}_{n=1}^{\infty},\left\{b_{\beta, \omega}(n)\right\}_{n=1}^{\infty}\right)$ is defined by:

(1) The random variables $\left\{a_{\beta, \omega}(n)\right\}_{n=1}^{\infty},\left\{b_{\beta, \omega}(n)\right\}_{n=1}^{\infty}$ are all independent.

(2) $b_{\beta, \omega}(n)$ are all standard Gaussian variables (that is, with zero mean and variance $=1)$, irrespective of $\beta$ and $n$.

(3) The probability distribution function of $a_{\beta, \omega}(n)$ is given by

$$
P\left\{\omega \mid a_{\beta, \omega}(n)<C\right\}=\frac{2}{\Gamma\left(\frac{\beta n}{2}\right)} \int_{0}^{C} x^{\beta n-1} e^{-x^{2}} d x .
$$

In 6, Dumitriu and Edelman showed that the eigenvalue distribution function of the finite matrix, obtained as the restriction of $J_{\beta, \omega}$ to the $N \times N$ upper left corner, is $f_{\beta, N}$ for any $\beta>0$.

From property (3) above, it follows that

$$
\begin{aligned}
\left\langle a_{\beta, \omega}(n)\right\rangle \equiv & \int_{\Omega} a_{\beta, \omega}(n) d \omega=\frac{\Gamma\left(\frac{\beta n+1}{2}\right)}{\Gamma\left(\frac{\beta n}{2}\right)}=\sqrt{\frac{\beta n}{2}}\left(1-\frac{1}{4 \beta n}\right)+\mathcal{O}\left(\frac{1}{n^{\frac{3}{2}}}\right), \\
& \left\langle\left(a_{\beta, \omega}(n)-\left\langle a_{\beta, \omega}(n)\right\rangle\right)^{2}\right\rangle=\frac{1}{4}+\mathcal{O}\left(\frac{1}{n}\right) .
\end{aligned}
$$

Thus we see that the family $J_{\beta, \omega}$ corresponds to the case $\eta_{1}=1 / 2, \eta_{2}=0$ of the general matrices introduced above. Technically, the following theorem is not a 
corollary of Theorem 1.1 because of the $\mathcal{O}\left(n^{-1 / 2}\right)$ term in (1.10) and the $\mathcal{O}\left(n^{-1}\right)$ term in (1.11). The proof of Theorem 1.1. however, is robust with respect to such a change, and we have

Theorem 1.4. For any $\beta$, the spectrum of $J_{\beta, \omega}$ is $\mathbb{R}$ with probability one.

If $\beta<2$, then, with probability one, the spectral measure, $\mu_{\beta, \omega}$, corresponding to $J_{\beta, \omega}$ and $\delta_{1}$, is pure point with eigenfunctions decaying as

$$
\left|\psi_{\omega}(n)\right|^{2} \sim n^{-\left(\frac{1}{2}+\frac{1}{\beta}\right)} .
$$

If $\beta \geq 2$, then with probability one, for any $\varepsilon>0, \mu_{\beta, \omega}$ has exact dimension $1-\frac{2}{\beta}$ with probability one. Furthermore, for $\beta \geq 2$, we have that, almost surely,

$$
\frac{1}{T} \int_{0}^{T}\left|\left(\hat{X}^{m} e^{-i t J_{\beta, \omega}} \psi, e^{-i t J_{\beta, \omega}} \psi\right)\right| d t \geq C(\omega, \psi, m, \varepsilon) T^{2 m-\varepsilon}
$$

for any $\psi, \varepsilon>0$ and $m$.

This result, without the dynamical part, was announced in 2. We note that the analogous Circular $\beta$ Ensembles can be realized as eigenvalues of truncated CMV matrices. This was shown by Killip and Nenciu [10] and later used by Killip and Stoiciu in their analysis of level statistics for ensembles of random CMV matrices [11. The bulk spectral properties of the appropriate matrices were analyzed by Simon [21, Section 12.7].

The proof of Theorem 1.1]is given in the next section. Since the proof of the spectral part of Theorem 1.4 is precisely the same, it is not given separately. As noted earlier, the dynamical part of our analysis (Theorem 1.2 and the corresponding statement in Theorem (1.4) follows immediately from Theorem 1.1 and Proposition 2.7, and by Theorem 1.2 of [12.

The method we use is a variation on the one used by Kiselev-Last-Simon 13, Section 8] in their analysis of the Schrödinger case described above. A notable difference is the fact that, due to the growth of the $a(n)$, the effective energy parameter, $\frac{E}{a(n)}$, vanishes in the limit. This, in addition to requiring a modification in the technique of proof (see Lemma 2.4 and Proposition 2.5 below), leads to the fact that the asymptotics of the generalized eigenfunctions are constant over $\mathbb{R}$. At the critical point $\left(\left(\eta_{1}-\eta_{2}\right)=\frac{1}{2}\right)$, this implies uniformity of the local Hausdorff dimensions of the spectral measure.

A modified Combes-Thomas estimate, for operators with unbounded off-diagonal terms, enters our analysis in the identification of the spectrum of $J_{\Upsilon, \omega}$. Such an estimate may be of independent interest, and thus is presented in the Appendix.

\section{Proof of Theorem 1.1}

We begin with a simple lemma that shows that, in a certain sense, $J_{\Upsilon, \omega}$ is a random relatively decaying perturbation of $J_{\lambda_{1}, \eta_{1}}$.

Lemma 2.1. For any $\varepsilon>0$ there exists, with probability one, a constant $C=$ $C(\omega, \varepsilon)$ for which

$$
\left|\frac{\left|n^{\eta_{2}} X_{\omega}(n)\right|}{n^{\eta_{1}}}\right| \leq \frac{C}{n^{\gamma-\varepsilon}}
$$


and

$$
\left|\frac{\left|n^{\eta_{2}} Y_{\omega}(n)\right|}{n^{\eta_{1}}}\right| \leq \frac{C}{n^{\gamma-\varepsilon}}
$$

where $\gamma=\eta_{1}-\eta_{2}$.

Proof. By (1.3) and Chebyshev's inequality we have for any $k \in \mathbb{N}$

$$
P_{n} \equiv \mathcal{P}\left\{\omega|| \frac{\left|n^{\eta_{2}} X_{\omega}(n)\right|}{n^{\eta_{1}}} \mid \geq \frac{1}{n^{\gamma-\varepsilon}}\right\} \leq \frac{C(k)}{n^{2 k \varepsilon}} .
$$

By choosing $2 k>\varepsilon^{-1}$ we see that

$$
\sum_{n=1}^{\infty} P_{n}<\infty
$$

(2.1) now follows from the Borel-Cantelli Lemma. The proof of (2.2) is the same.

As stated in the Introduction, we follow the strategy of [13. In particular, we will deduce the spectral properties of $J_{\Upsilon, \omega}$ from the asymptotics of the solutions to the corresponding eigenfunction equation.

In order to fix notation, for a given Jacobi matrix $J\left(\{a(n)\}_{n=1}^{\infty},\{b(n)\}_{n=1}^{\infty}\right)$ and a fixed $E \in \mathbb{R}$, denote by $\psi^{E}$ a solution to the equation

$$
a(n) \psi^{E}(n+1)+b(n) \psi^{E}(n)+a(n-1) \psi^{E}(n-1)=E \psi^{E}(n), \quad n>1 .
$$

It is customary to extend this equation to $n=1$ by defining $a(0)=1$. Clearly, the space of sequences $\left\{\psi^{E}(n)\right\}_{n=0}^{\infty}$ solving (2.3) is a two-dimensional vector space, and any such sequence is completely determined by its values at 0 and 1 . We let $\psi_{\phi}^{E}(n)$ stand for the solution of (2.3) satisfying

$$
\psi_{\phi}^{E}(0)=\sin (\phi), \quad \psi_{\phi}^{E}(1)=\cos (\phi) .
$$

We note that, formally, $\psi_{0}^{E}(n)$ satisfies

$$
J \psi_{0}^{E}=E \psi_{0}^{E} .
$$

Let

$$
S^{E}(n)=\left(\begin{array}{cc}
\frac{E-b(n)}{a(n)} & -\frac{a(n-1)}{a(n)} \\
1 & 0
\end{array}\right)
$$

and

$$
T^{E}(n)=S^{E}(n) \cdot S^{E}(n-1) \cdots S^{E}(1) .
$$

Then, for any $\phi$,

$$
\left(\begin{array}{c}
\psi_{\phi}^{E}(n+1) \\
\psi_{\phi}^{E}(n)
\end{array}\right)=T^{E}(n)\left(\begin{array}{c}
\psi_{\phi}^{E}(1) \\
\psi_{\phi}^{E}(0)
\end{array}\right)
$$

and so

$$
T^{E}(n)=\left(\begin{array}{cc}
\psi_{0}^{E}(n+1) & \psi_{\frac{\pi}{2}}^{E}(n+1) \\
\psi_{0}^{E}(n) & \psi_{\frac{\pi}{2}}^{E}(n)
\end{array}\right) .
$$

We call the matrices $S^{E}(n)$ defined above one-step transfer matrices, and for the matrices $T^{E}(n)$ we use the name $n$-step transfer matrices. Our main technical result is

Theorem 2.2. Let $J_{\Upsilon, \omega}$ be the family of random Jacobi matrices described in the Introduction. Then, for any $E \in \mathbb{R}$, the following holds with probability one. 
(1) If $\gamma>\frac{1}{2}$

$$
\lim _{n \rightarrow \infty} \frac{\log \left\|T_{\omega}^{E}(n)\right\|^{2}}{\log (n)}=-\eta_{1}
$$

(2) If $\gamma=\frac{1}{2}$

$$
\lim _{n \rightarrow \infty} \frac{\log \left\|T_{\omega}^{E}(n)\right\|^{2}}{\log (n)}=\Lambda-\eta_{1}
$$

(3) If $\gamma<\frac{1}{2}$

$$
\lim _{n \rightarrow \infty} \frac{\log \left\|T_{\omega}^{E}(n)\right\|^{2}}{n^{1-2 \gamma}}=\Lambda .
$$

The EFGP transform (see [13) is a useful tool for studying the asymptotic behavior of $\left\|T^{E}(n)\right\|$ in the Schrödinger case $(a(n) \equiv 1)$. For $a(n) \rightarrow \infty$, certain modifications are needed. We proceed to present a version that is suitable for our purposes.

Let $J\left(\left\{a_{\omega}(n)\right\}_{n=1}^{\infty}\left\{b_{\omega}(n)\right\}_{n=1}^{\infty}\right)$ be a Jacobi matrix whose entries are all independent random variables. Let $\tilde{a}(n)=\left\langle a_{\omega}(n)\right\rangle$ and $\alpha_{\omega}=a_{\omega}(n)-\tilde{a}(n)$, and assume that

$$
\lim _{n \rightarrow \infty} \tilde{a}(n)=\infty
$$

and that

$$
\lim _{n \rightarrow \infty} \frac{\alpha_{\omega}(n)}{\tilde{a}(n)}=0
$$

with probability one. These properties clearly hold for $J_{\Upsilon, \omega}$ (see Lemma 2.1). In the analysis that follows we keep $E \in \mathbb{R}$ fixed, so we omit it from the notation. Define

Then,

$$
K_{\omega}(n)=\left(\begin{array}{cc}
1 & 0 \\
0 & a_{\omega}(n)
\end{array}\right) .
$$

$$
\tilde{S}_{\omega}(n) \equiv K_{\omega}(n) S(n) K_{\omega}(n-1)^{-1}=\left(\begin{array}{cc}
\frac{E-b_{\omega}(n)}{a_{\omega}(n)} & -\frac{1}{a_{\omega}(n)} \\
a_{\omega}(n) & 0
\end{array}\right)
$$

and

$$
\tilde{T}_{\omega}(n) \equiv \tilde{S}_{\omega}(n) \cdot \tilde{S}_{\omega}(n-1) \cdots \tilde{S}_{\omega}(1)=K_{\omega}(n) T_{\omega}(n) .
$$

For any $\phi$, define the sequences $\left\{u_{\omega, \phi}(n)\right\}_{n=1}^{\infty}$ and $\left\{v_{\omega, \phi}(n)\right\}_{n=1}^{\infty}$ by

$$
\left(\begin{array}{c}
u_{\omega, \phi}(n) \\
v_{\omega, \phi}(n)
\end{array}\right)=\tilde{T}_{\omega}(n)\left(\begin{array}{c}
\cos (\phi) \\
\sin (\phi)
\end{array}\right) \text {, }
$$

so that, from the definition of $\psi_{\omega, \phi}\left(=\psi_{\phi}\right.$ for the random Jacobi parameters), we see that

$$
u_{\omega, \phi}(n)=\psi_{\omega, \phi}(n+1), \quad v_{\omega, \phi}(n)=a_{\omega}(n) \psi_{\omega, \phi}(n) .
$$

By (2.8) we see that for any $E \in \mathbb{R}$ and sufficiently large $n$, we may define $k_{n} \in(0, \pi)$ by

$$
2 \cos \left(k_{n}\right)=\frac{E}{\tilde{a}(n)} .
$$

Clearly, $k_{n} \rightarrow \frac{\pi}{2}$ as $n \rightarrow \infty$. 
Now, define $R_{\omega, \phi}(n)$ and $\theta_{\omega, \phi}(n)$ through

$$
R_{\omega, \phi}(n) \sin \left(\theta_{\omega, \phi}(n)\right)=v_{\omega, \phi}(n) \sin \left(k_{n}\right)
$$

and

$$
R_{\omega, \phi}(n) \cos \left(\theta_{\omega, \phi}(n)\right)=\tilde{a}(n) u_{\omega, \phi}(n)-v_{\omega, \phi}(n) \cos \left(k_{n}\right),
$$

so that (using (2.10)

$$
\begin{aligned}
R_{\omega, \phi}(n)^{2}= & v_{\omega, \phi}(n)^{2}+\tilde{a}(n)^{2} u_{\omega, \phi}(n)^{2}-2 \tilde{a}(n) u_{\omega, \phi}(n) v_{\omega, \phi}(n) \cos \left(k_{n}\right) \\
= & a_{\omega}(n)^{2} \psi_{\omega, \phi}(n)^{2}+\tilde{a}(n)^{2} \psi_{\omega, \phi}(n+1)^{2} \\
& -a_{\omega}(n) E \psi_{\omega, \phi}(n+1) \psi_{\omega, \phi}(n)
\end{aligned}
$$

which leads to

$$
\begin{aligned}
\frac{R_{\omega, \phi}(n)^{2}}{\tilde{a}(n)^{2}\left(\psi_{\omega, \phi}(n)^{2}+\psi_{\omega, \phi}(n+1)^{2}\right)}= & 1+\frac{2 \alpha_{\omega}(n) \tilde{a}(n) \psi_{\omega, \phi}(n)^{2}}{\tilde{a}(n)^{2}\left(\psi_{\omega, \phi}(n)^{2}+\psi_{\omega, \phi}(n+1)^{2}\right)} \\
& +\frac{2 \alpha_{\omega}(n)^{2} \psi_{\omega, \phi}(n)^{2}}{\tilde{a}(n)^{2}\left(\psi_{\omega, \phi}(n)^{2}+\psi_{\omega, \phi}(n+1)^{2}\right)} \\
& -\frac{E a_{\omega}(n) \psi_{\omega, \phi}(n) \psi_{\omega, \phi}(n+1)}{\tilde{a}(n)^{2}\left(\psi_{\omega, \phi}(n)^{2}+\psi_{\omega, \phi}(n+1)^{2}\right)} .
\end{aligned}
$$

By (2.9), it follows that the right hand side converges to one with probability one, uniformly in $\phi$, so that almost surely, for sufficiently large $n$, there are constants $C_{1}, C_{2}>0$ such that

$$
C_{1} R_{\omega, \phi}(n)^{2} \leq \tilde{a}(n)^{2}\left(\psi_{\omega, \phi}(n)^{2}+\psi_{\omega, \phi}(n+1)^{2}\right) \leq C_{2} R_{\omega, \phi}(n)^{2} .
$$

Now, by a straightforward adaptation of Lemma 2.2 of [13], it follows that for any two angles $\phi_{1} \neq \phi_{2}$, there are constants $C_{3}, C_{4}>0$ such that

$$
\begin{aligned}
C_{3} \max \left(R_{\omega, \phi_{1}}(n)^{2}, R_{\omega, \phi_{2}}(n)^{2}\right) & \leq \tilde{a}(n)^{2}\left\|T_{\omega}(n)\right\|^{2} \\
& \leq C_{4} \max \left(R_{\omega, \phi_{1}}(n)^{2}, R_{\omega, \phi_{2}}(n)^{2}\right) .
\end{aligned}
$$

Thus, we are led to examine the asymptotic properties of $\log R_{\omega, \phi}(n)$.

Let us formulate a recursion relation for $R_{\omega, \phi}(n)^{2}:(2.12)$ and (2.13) mean

$$
\begin{aligned}
& \left(\begin{array}{c}
R_{\omega, \phi}(n) \sin \left(\theta_{\omega, \phi}(n)\right) \\
R_{\omega, \phi}(n) \cos \left(\theta_{\omega, \phi}(n)\right)
\end{array}\right) \\
& =\left(\begin{array}{cc}
0 & \sin \left(k_{n}\right) \\
\tilde{a}(n) & -\cos \left(k_{n}\right)
\end{array}\right)\left(\begin{array}{c}
u_{\omega, \phi}(n) \\
v_{\omega, \phi}(n)
\end{array}\right) \\
& =\left(\begin{array}{cc}
0 & \sin \left(k_{n}\right) \\
\tilde{a}(n) & -\cos \left(k_{n}\right)
\end{array}\right)\left(\begin{array}{cc}
1 & 0 \\
0 & a_{\omega}(n)
\end{array}\right)\left(\begin{array}{c}
\psi_{\omega, \phi}(n+1) \\
\psi_{\omega, \phi}(n)
\end{array}\right) .
\end{aligned}
$$

We also know that

$$
\left(\begin{array}{c}
\psi_{\omega, \phi}(n+2) \\
\psi_{\omega, \phi}(n+1)
\end{array}\right)=S_{\omega}(n+1)\left(\begin{array}{c}
\psi_{\omega, \phi}(n+1) \\
\psi_{\omega, \phi}(n)
\end{array}\right),
$$


SO

$$
\begin{aligned}
&\left(\begin{array}{c}
R_{\omega, \phi}(n+1) \sin \left(\theta_{\omega, \phi}(n+1)\right) \\
R_{\omega, \phi}(n+1) \cos \left(\theta_{\omega, \phi}(n+1)\right)
\end{array}\right) \\
&=\left(\begin{array}{cc}
0 & \sin \left(k_{n+1}\right) \\
\tilde{a}(n+1) & -\cos \left(k_{n+1}\right)
\end{array}\right)\left(\begin{array}{cc}
1 & 0 \\
0 & a_{\omega}(n+1)
\end{array}\right) S_{\omega}(n+1) \\
& \\
& \cdot\left(\begin{array}{cc}
1 & 0 \\
0 & a_{\omega}(n)
\end{array}\right)^{-1}\left(\begin{array}{cc}
0 & \sin \left(k_{n}\right) \\
\tilde{a}(n) & -\cos \left(k_{n}\right)
\end{array}\right)\left(\begin{array}{c}
R_{\omega, \phi}(n) \sin \left(\theta_{\omega, \phi}(n)\right) \\
R_{\omega, \phi}(n) \cos \left(\theta_{\omega, \phi}(n)\right)
\end{array}\right) \\
&=\left(\begin{array}{cc}
0 & \sin \left(k_{n+1}\right) \\
\tilde{a}(n+1) & -\cos \left(k_{n+1}\right)
\end{array}\right) \tilde{S}_{\omega}(n+1) \\
& \cdot\left(\begin{array}{cc}
0 & \sin \left(k_{n}\right) \\
\tilde{a}(n) & -\cos \left(k_{n}\right)
\end{array}\right)^{-1}\left(\begin{array}{l}
R_{\omega, \phi}(n) \sin \left(\theta_{\omega, \phi}(n)\right) \\
R_{\omega, \phi}(n) \cos \left(\theta_{\omega, \phi}(n)\right)
\end{array}\right) .
\end{aligned}
$$

Now, write

$$
\begin{aligned}
\tilde{S}_{\omega}(n+1)= & \left(\begin{array}{cc}
\frac{E-b_{\omega}(n+1)}{a_{\omega}(n+1)} & -\frac{1}{a_{\omega}(n+1)} \\
a_{\omega}(n+1) & 0
\end{array}\right) \\
= & \frac{\tilde{a}(n+1)}{a_{\omega}(n+1)}\left(\left(\begin{array}{cc}
\frac{E}{\tilde{a}(n+1)} & -\frac{1}{\tilde{a}(n+1)} \\
\tilde{a}(n+1) & 0
\end{array}\right)\right. \\
& \left.+\left(\begin{array}{cc}
-\frac{b_{\omega}(n+1)}{\tilde{a}(n+1)} & 0 \\
\frac{a_{\omega}(n+1)^{2}-\tilde{a}(n+1)^{2}}{\tilde{a}(n+1)} & 0
\end{array}\right)\right) .
\end{aligned}
$$

We define

$$
\begin{aligned}
\mathcal{Z}_{\omega}(n+1)= & \left(\begin{array}{cc}
0 & \sin \left(k_{n+1}\right) \\
\tilde{a}(n+1) & -\cos \left(k_{n+1}\right)
\end{array}\right)\left(\begin{array}{cc}
\frac{E}{\tilde{a}(n+1)} & -\frac{1}{\tilde{a}(n+1)} \\
\tilde{a}(n+1) & 0
\end{array}\right) \\
& \cdot\left(\begin{array}{cc}
0 & \sin \left(k_{n}\right) \\
\tilde{a}(n) & -\cos \left(k_{n}\right)
\end{array}\right)^{-1}\left(\begin{array}{c}
\sin \left(\theta_{\omega, \phi}(n)\right) \\
\cos \left(\theta_{\omega, \phi}(n)\right)
\end{array}\right)
\end{aligned}
$$

and

$$
\begin{aligned}
\mathcal{W}_{\omega}(n+1)= & \left(\begin{array}{cc}
0 & \sin \left(k_{n+1}\right) \\
\tilde{a}(n+1) & -\cos \left(k_{n+1}\right)
\end{array}\right)\left(\begin{array}{cc}
-\frac{b_{\omega}(n+1)}{\tilde{a}(n+1)} & 0 \\
\frac{a_{\omega}(n+1)^{2}-\tilde{a}(n+1)^{2}}{\tilde{a}(n+1)} & 0
\end{array}\right) \\
& \cdot\left(\begin{array}{cc}
0 & \sin \left(k_{n}\right) \\
\tilde{a}(n) & -\cos \left(k_{n}\right)
\end{array}\right)^{-1}\left(\begin{array}{c}
\sin \left(\theta_{\omega, \phi}(n)\right) \\
\cos \left(\theta_{\omega, \phi}(n)\right)
\end{array}\right)
\end{aligned}
$$

(we ignore the dependence on $\phi$ since we keep it fixed). Then, from (2.16) we see that

$$
\frac{R_{\omega, \phi}(n+1)^{2}}{R_{\omega, \phi}(n)^{2}}=\frac{\tilde{a}(n+1)^{2}}{a_{\omega}(n+1)^{2}}\left\|\mathcal{Z}_{\omega}(n+1)+\mathcal{W}_{\omega}(n+1)\right\|^{2}
$$


$\theta_{\omega, \phi}(n)$ satisfies a recurrence relation as well: From (2.10) we have that

$$
v_{\omega, \phi}(n+1)=a_{\omega}(n+1) u_{\omega, \phi}(n)
$$

and

$$
a_{\omega}(n+1) u_{\omega, \phi}(n+1)+b_{\omega}(n+1) u_{\omega, \phi}(n)+a_{\omega}(n) u_{\omega, \phi}(n-1)=E u_{\omega, \phi}(n) .
$$

Write, using (2.12)-(2.13), that

$$
\begin{aligned}
\cot \left(\theta_{\omega, \phi}(n+1)\right) & =\frac{\tilde{a}(n+1) u_{\omega, \phi}(n+1)-\cos \left(k_{n+1}\right) v_{\omega, \phi}(n+1)}{\sin \left(k_{n+1}\right) v_{\omega, \phi}(n+1)} \\
& =\frac{\tilde{a}(n+1) u_{\omega, \phi}(n+1)-a_{\omega}(n+1) \cos \left(k_{n+1}\right) u_{\omega, \phi}(n)}{\sin \left(k_{n+1}\right) a_{\omega}(n+1) u_{\omega, \phi}(n)} .
\end{aligned}
$$

Furthermore, observing that

$$
R_{\omega, \phi}(n) \sin \left(\theta_{\omega, \phi}(n)+k_{n}\right)=\tilde{a}(n) \sin \left(k_{n}\right) u_{\omega, \phi}(n)
$$

and

$$
\begin{aligned}
R_{\omega, \phi}(n) \cos \left(\theta_{\omega, \phi}(n)+k_{n}\right) & =\tilde{a}(n) \cos \left(k_{n}\right) u_{\omega, \phi}(n)-v_{\omega, \phi}(n) \\
& =\tilde{a}(n) \cos \left(k_{n}\right) u_{\omega, \phi}(n)-a_{\omega}(n) u_{\omega, \phi}(n-1),
\end{aligned}
$$

we may write

$$
\cot \left(\theta_{\omega, \phi}(n)+k_{n}\right)=\frac{\tilde{a}(n) \cos \left(k_{n}\right) u_{\omega, \phi}(n)-a_{\omega}(n) u_{\omega, \phi}(n-1)}{\tilde{a}(n) \sin \left(k_{n}\right) u_{\omega, \phi}(n)} .
$$

Substituting $u_{\omega, \phi}(n+1)$ from (2.19) into (2.20) and then $u_{\omega, \phi}(n-1)$ from (2.21) into the resulting equation, we get

$$
\begin{aligned}
\cot \left(\theta_{\omega, \phi}(n+1)\right)= & \frac{\tilde{a}(n+1) \tilde{a}(n)}{a_{\omega}(n+1)^{2}} \frac{\sin \left(k_{n}\right)}{\sin \left(k_{n+1}\right)} \cot \left(\theta_{\omega, \phi}(n)+k_{n}\right) \\
& +\cot \left(k_{n+1}\right)\left(\frac{\tilde{a}(n+1)^{2}}{a_{\omega}(n+1)^{2}}-1\right) \\
& -\frac{\tilde{a}(n+1)}{\sin \left(k_{n}\right) a_{\omega}(n+1)^{2}} b_{\omega}(n+1) \\
\equiv & \kappa_{\omega}(n+1) \cot \left(\bar{\theta}_{\omega, \phi}(n)\right)+\zeta_{\omega}(n+1),
\end{aligned}
$$

where

$$
\bar{\theta}_{\omega, \phi}(n)=\theta_{\omega, \phi}(n)+k_{n} .
$$

By (2.15) and picking two angles $\phi_{1} \neq \phi_{2}$, Theorem 2.2 follows from

Proposition 2.3. Let $J_{\Upsilon, \omega}$ be the family of random Jacobi matrices described in the Introduction. Then, for any $E \in \mathbb{R}$ and for any $\phi$, the following holds with probability one:

(1) If $\gamma>\frac{1}{2}$

$$
\lim _{n \rightarrow \infty} \frac{\log R_{\omega, \phi}^{E}(n)^{2}}{\log (n)}=\eta_{1}
$$

(2) If $\gamma=\frac{1}{2}$

$$
\lim _{n \rightarrow \infty} \frac{\log R_{\omega, \phi}^{E}(n)^{2}}{\log (n)}=\Lambda+\eta_{1}
$$


(3) If $\gamma<\frac{1}{2}$

$$
\lim _{n \rightarrow \infty} \frac{\log R_{\omega, \phi}^{E}(n)^{2}}{n^{1-2 \gamma}}=\Lambda .
$$

Proof. As in [13] we shall prove the statement by using the recursion relation for $R_{\omega}(n)^{2}$ (equation (2.17)). Namely, we shall prove that

$$
\frac{1}{F_{\gamma}(n)} \sum_{j=1}^{n}\left(\log \left(\left\|\mathcal{Z}_{\omega}(j)+\mathcal{W}_{\omega}(j)\right\|^{2}\right)-\log \left(\frac{a_{\omega}(j)^{2}}{\tilde{a}(j)^{2}}\right)\right)
$$

converges to the appropriate limit, where $F_{\gamma}(n)=\log (n)$ for $\gamma \geq \frac{1}{2}$ and $F_{\gamma}(n)=$ $n^{1-2 \gamma}$ otherwise. From this point on, $a_{\omega}(n)=a_{\Upsilon, \omega}(n)$ and $b_{\omega}(n)=b_{\Upsilon, \omega}(n)$.

We shall need some estimate on the behavior of $\theta_{\omega}(n)$. We start with

Lemma 2.4. For any $\varepsilon>0$, there exists, with probability one, a constant $\tilde{C}=$ $\tilde{C}(\omega, \varepsilon)$, such that

$$
\left|\theta_{\omega, \phi}(n+1)-\bar{\theta}_{\omega, \phi}(n)\right| \leq \tilde{C} \max \left(n^{-\gamma+\varepsilon}, n^{-1}\right) .
$$

Proof of Lemma 2.4. We start by proving a similar statement for $\left|\kappa_{\omega}(n+1)-1\right|+$ $\left|\zeta_{\omega}(n+1)\right|:$

Recall that $k_{n} \rightarrow \frac{\pi}{2}$. Thus, for large enough $n, \sin \left(k_{n}\right)>\frac{1}{2}$. Moreover, $\cos \left(k_{n}\right) \sim$ $\frac{1}{n^{\eta_{1}}}$. It follows that

$$
\left|\sin \left(k_{n}\right)-\sin \left(k_{n+1}\right)\right|=\frac{\left|\cos \left(k_{n}\right)^{2}-\cos \left(k_{n+1}\right)^{2}\right|}{\left|\sin \left(k_{n}\right)+\sin \left(k_{n+1}\right)\right|} \leq \frac{C^{\prime}}{n^{1+2 \eta_{1}}} .
$$

Thus,

$$
\begin{aligned}
\left|\kappa_{\omega}(n+1)-1\right|+\left|\zeta_{\omega}(n+1)\right| \leq & \left|\frac{\sin \left(k_{n}\right)}{\sin \left(k_{n+1}\right)}\left(\frac{\tilde{a}(n+1) \tilde{a}(n)}{a_{\omega}(n+1)^{2}}-1\right)\right| \\
& +\left|\frac{\sin \left(k_{n}\right)-\sin \left(k_{n+1}\right)}{\sin \left(k_{n+1}\right)}\right| \\
& +\left|\cot \left(k_{n+1}\right)\left(\frac{\tilde{a}(n+1)^{2}}{a_{\omega}(n+1)^{2}}-1\right)\right| \\
& +\left|\frac{\tilde{a}(n+1)}{\sin \left(k_{n}\right) a_{\omega}(n+1)^{2}} b_{\omega}(n+1)\right| \\
= & I_{1}+I_{2}+I_{3}+I_{4} .
\end{aligned}
$$

Now, choosing $\varepsilon<\eta_{1}-\eta_{2}$, by Lemma 2.1

$$
\begin{array}{r}
I_{1} \leq 2\left|\frac{\tilde{a}(n+1)(\tilde{a}(n)-\tilde{a}(n+1))}{a_{\omega}(n+1)^{2}}\right|+4\left|\frac{\alpha_{\omega}(n+1) \tilde{a}(n+1)}{a_{\omega}(n+1)^{2}}\right| \\
+2\left|\frac{\alpha_{\omega}(n+1)^{2}}{a_{\omega}(n+1)^{2}}\right| \leq C_{1}(\omega, \varepsilon) \max \left(n^{-\gamma+\varepsilon}, n^{-1}\right), \\
I_{3} \leq 2\left|\cos \left(k_{n+1}\right)\right|\left|\frac{2 \alpha_{\omega}(n+1) \tilde{a}(n+1)+\alpha_{\omega}(n+1)^{2}}{a_{\omega}(n+1)^{2}}\right| \leq \frac{C_{3}(\omega, \varepsilon)}{n^{\gamma-\varepsilon}},
\end{array}
$$


and

almost surely. We have shown above that

$$
I_{4} \leq 2\left|\frac{\tilde{a}(n+1) b_{\omega}(n+1)}{a_{\omega}(n+1)^{2}}\right| \leq \frac{C_{4}(\omega, \varepsilon)}{n^{\gamma-\varepsilon}}
$$

$$
I_{2} \leq \frac{C_{2}}{n^{1+\eta_{1}}}
$$

so we see that there exists, with probability one, a constant $C_{5}(\omega, \varepsilon)$ for which

$$
\left|\kappa_{\omega}(n+1)-1\right|+\left|\zeta_{\omega}(n+1)\right| \leq \frac{C_{5}}{\max }\left(n^{-\gamma+\varepsilon}, n^{-1}\right)
$$

Now use

$$
e^{2 i x}=1-\frac{2}{1+i \cot x}
$$

and (2.22) to see that, if $\left|\kappa_{\omega}(n+1)-1\right|+2\left|\zeta_{\omega}(n+1)\right|<\frac{1}{2}$ (which indeed happens almost surely, for large enough $n$ ), then

$$
\left|e^{2 i \theta_{\omega, \phi}(n+1)}-e^{2 i \bar{\theta}_{\omega, \phi}(n)}\right| \leq 4\left(\left|\kappa_{\omega}(n+1)-1\right|+\left|\zeta_{\omega}(n+1)\right|\right),
$$

which implies (by $\left|e^{i x}-1\right| \geq \frac{2|x|}{\pi}$ ) that

$$
\left|\theta_{\omega, \phi}(n+1)-\bar{\theta}_{\omega, \phi}(n)\right| \leq \pi\left(\left|\kappa_{\omega}(n+1)-1\right|+\left|\zeta_{\omega}(n+1)\right|\right) .
$$

This, together with (2.28), implies (2.27) and concludes the proof of the lemma.

The direct consequence of this is

Proposition 2.5. Assume $f(n)$ is a function that satisfies

$$
f(n)=o\left(n^{r}\right)
$$

and

with

$$
f(n+1)-f(n)=o\left(n^{r-1}\right),
$$

$$
r= \begin{cases}\gamma-1 & \text { if } \gamma \geq 1 / 2 \text { and } \eta_{2}>0 \\ -\gamma & \text { if } \gamma<1 / 2 \text { and } \eta_{2}>0 \\ \eta_{1}-1 & \text { if } \gamma \geq 1 / 2 \text { and } \eta_{2} \leq 0 \\ \eta_{1}-2 \gamma & \text { if } \gamma<1 / 2 \text { and } \eta_{2} \leq 0\end{cases}
$$

Then, for any $\phi$,

$$
\lim _{n \rightarrow \infty} \frac{1}{F_{\gamma}(n)} \sum_{j=1}^{n} f(j) \cos \left(2 \theta_{\omega, \phi}(j)\right)=0
$$

almost surely. The same statement holds with $\theta$ replaced by $\bar{\theta}$.

Proof of Proposition 2.5. We shall prove the statement for $\theta$. By summation by parts,

$$
\begin{aligned}
& \left|\sum_{j=1}^{n} f(j) \cos \left(2 \theta_{\omega, \phi}(j)\right)\right| \\
& \quad=\left|f(n) \sum_{j=1}^{n} \cos \left(2 \theta_{\omega, \phi}(j)\right)-\sum_{j=1}^{n-1} \sum_{l=1}^{j} \cos \left(2 \theta_{\omega, \phi}(l)\right)(f(j+1)-f(j))\right| .
\end{aligned}
$$


Thus we are led to examine $\sum_{l=1}^{j} \cos \left(2 \theta_{\omega, \phi}(l)\right)$. Assume that $j=2 m$ is even. Then

$$
\begin{aligned}
\left|\sum_{l=1}^{j} \cos \left(2 \theta_{\omega, \phi}(l)\right)\right| & =\left|\sum_{l=1}^{m}\left(\cos \left(2 \theta_{\omega, \phi}(2 l)\right)-\cos \left(2 \theta_{\omega, \phi}(2 l-1)+\pi\right)\right)\right| \\
& \leq \sum_{l=1}^{m}\left|2 \theta_{\omega, \phi}(2 l)-2 \theta_{\omega, \phi}(2 l-1)-\pi\right| \\
& \leq 2 \sum_{l=1}^{m}\left|\theta_{\omega, \phi}(2 l)-\bar{\theta}_{\omega, \phi}(2 l-1)\right|+\left|k_{2 l-1}-\frac{\pi}{2}\right| \\
& \leq C_{\omega} \max \left(j^{1-\gamma+\varepsilon}, j^{1-\eta_{1}}\right)
\end{aligned}
$$

almost surely, by Lemma 2.4 and by

$$
\left|k_{n}-\frac{\pi}{2}\right| \leq 2\left|\cos \left(k_{n}\right)\right|,
$$

which holds for sufficiently large $n$. Thus, for any $j$, we get

$$
\begin{aligned}
\left|\sum_{l=1}^{j} \cos \left(2 \theta_{\omega, \phi}(l)\right)\right| & \leq C_{\omega} \max \left(j^{1-\gamma+\varepsilon}, j^{1-\eta_{1}}\right)+1 \\
& \leq\left(C_{\omega}+1\right) \max \left(j^{1-\gamma+\varepsilon}, j^{1-\eta_{1}}\right)
\end{aligned}
$$

A simple calculation finishes the proof for $\theta$. The proof for $\bar{\theta}$ follows the same argument, with an additional $\left|k_{2 l}-k_{2 l-1}\right|$ term in (2.32).

We abbreviate

$$
\begin{aligned}
A_{\omega}(j) & =\frac{a_{\omega}(j)^{2}-\tilde{a}(j)^{2}}{\tilde{a}(j)^{2}}=2 \frac{\alpha_{\omega}(j)}{\tilde{a}(j)}+\frac{\alpha_{\omega}(j)^{2}}{\tilde{a}(j)^{2}} \\
& =2 \frac{\lambda_{2}}{\lambda_{1}} \frac{Y_{\omega}(j)}{j^{\gamma}}+\left(\frac{\lambda_{2}}{\lambda_{1}}\right)^{2} \frac{Y_{\omega}(j)^{2}}{j^{2 \gamma}}
\end{aligned}
$$

and

$$
B_{\omega}(j)=\frac{b_{\omega}(j)}{\tilde{a}(j)}=\frac{\lambda_{2}}{\lambda_{1}} \frac{X_{\omega}(j)}{j^{\gamma}} .
$$

By a straightforward calculation we get

$$
\begin{aligned}
\left\|\mathcal{W}_{\omega}(j)\right\|^{2}= & \frac{\sin ^{2}\left(\bar{\theta}_{\omega}(j-1)\right) \tilde{a}(j)^{2}}{\sin ^{2}\left(k_{j-1}\right) \tilde{a}(j-1)^{2}} \\
& \times\left(A_{\omega}(j)^{2}+B_{\omega}(j)^{2}+2 \cos \left(k_{j}\right) B_{\omega}(j) A_{\omega}(j)\right), \\
\left\|\mathcal{Z}_{\omega}(j)\right\|^{2}= & 1+\sin ^{2}\left(\bar{\theta}_{\omega}(j-1)\right) \frac{\tilde{a}(j)^{2}-\tilde{a}(j-1)^{2}}{\tilde{a}(j-1)^{2}} \\
& +\frac{\tilde{a}(j)^{2} \sin ^{2}\left(\bar{\theta}_{\omega}(j-1)\right)}{\tilde{a}(j-1)^{2}} \frac{\sin ^{2}\left(k_{j}\right)-\sin ^{2}\left(k_{j-1}\right)}{\sin ^{2}\left(k_{j-1}\right)}
\end{aligned}
$$


and

$$
\begin{aligned}
\left(\mathcal{Z}_{\omega}(j), \mathcal{W}_{\omega}(j)\right)= & \frac{\tilde{a}(j)}{\tilde{a}(j-1)} \\
& \times\left(\frac { A _ { \omega } ( j ) \operatorname { s i n } ( \overline { \theta } _ { \omega } ( j - 1 ) ) } { \operatorname { s i n } ^ { 2 } ( k _ { j - 1 } ) } \left(\frac{\tilde{a}(j)}{\tilde{a}(j-1)} \sin \left(\bar{\theta}_{\omega}(j-1)\right)\right.\right. \\
- & \left.\left.\cos \left(k_{j}\right) \sin \left(\theta_{\omega}(j-1)\right)\right)-\frac{B_{\omega}(j) \sin \left(2 \bar{\theta}_{\omega}(j-1)\right)}{2 \sin \left(k_{j-1}\right)}\right) .
\end{aligned}
$$

$\left(\right.$ Recall $\left.\bar{\theta}_{\omega}(j) \equiv \bar{\theta}_{\omega, \phi}(j)=\theta_{\omega, \phi}(j)+k_{j} \equiv \theta_{\omega}(j)+k_{j}.\right)$

Let $\varepsilon<\frac{\gamma}{8}$. By Lemma 2.1, the fact that $\cos \left(k_{j}\right) \sim \frac{1}{\tilde{a}(j)}$ and the identity

$$
\left|\sin ^{2}(x)-\sin ^{2}(y)\right|=\left|\cos ^{2}(x)-\cos ^{2}(y)\right|,
$$

it follows that $\left\|\mathcal{W}_{\omega}(j)\right\|^{2}=\mathcal{O}_{\omega}\left(j^{-2 \gamma+2 \varepsilon}\right),\left(\mathcal{Z}_{\omega}(j), \mathcal{W}_{\omega}(j)\right)=\mathcal{O}_{\omega}\left(j^{-\gamma+\varepsilon}\right)$ and $\left(\left\|\mathcal{Z}_{\omega}(j)\right\|^{2}-1\right)=\mathcal{O}\left(j^{-1}\right)$ with probability one, where the notation $\mathcal{O}_{\omega}$ indicates that the implicit constant depends on $\omega$. Thus, we can use

$$
\log (1+x)=x-\frac{x^{2}}{2}+\mathcal{O}\left(x^{3}\right)
$$

together with the observation that (almost surely)

$$
\begin{aligned}
& \left(\left\|\mathcal{Z}_{\omega}(j)\right\|^{2}-1+2\left(\mathcal{Z}_{\omega}(j), \mathcal{W}_{\omega}(j)\right)+\left\|\mathcal{W}_{\omega}(j)\right\|^{2}\right)^{2} \\
& \quad=4\left(\mathcal{Z}_{\omega}(j), \mathcal{W}_{\omega}(j)\right)^{2}+\mathcal{O}_{\omega}\left(\frac{1}{j^{3 \gamma-\varepsilon}}\right),
\end{aligned}
$$

to see that, with probability one, for large enough $j$ we have that

$$
\begin{aligned}
\log & \left(1+\left\|\mathcal{Z}_{\omega}(j)\right\|^{2}-1+2\left(\mathcal{Z}_{\omega}(j), \mathcal{W}_{\omega}(j)\right)+\left\|\mathcal{W}_{\omega}(j)\right\|^{2}\right) \\
= & \left(\left\|\mathcal{Z}_{\omega}(j)\right\|^{2}-1\right)+2\left(\mathcal{Z}_{\omega}(j), \mathcal{W}_{\omega}(j)\right)+\left\|\mathcal{W}_{\omega}(j)\right\|^{2} \\
& -2\left(\mathcal{Z}_{\omega}(j), \mathcal{W}_{\omega}(j)\right)^{2}+\mathcal{O}_{\omega}\left(\frac{1}{j^{3 \gamma-\varepsilon}}+\frac{1}{j^{1+\gamma-\varepsilon}}\right) .
\end{aligned}
$$

Therefore, since the addition of a finite number of terms is inconsequential, it follows that

$$
\begin{aligned}
\lim _{n \rightarrow \infty} & \frac{1}{F_{\gamma}(n)} \sum_{j=1}^{n} \log \left(\left\|\mathcal{Z}_{\omega}(j)+\mathcal{W}_{\omega}(j)\right\|^{2}\right) \\
= & \lim _{n \rightarrow \infty} \frac{1}{F_{\gamma}(n)} \sum_{j=1}^{n}\left(\left(\left\|\mathcal{Z}_{\omega}(j)\right\|^{2}-1\right)+2\left(\mathcal{Z}_{\omega}(j), \mathcal{W}_{\omega}(j)\right)+\left\|\mathcal{W}_{\omega}(j)\right\|^{2}\right. \\
& \left.-2\left(\mathcal{Z}_{\omega}(j), \mathcal{W}_{\omega}(j)\right)^{2}\right)
\end{aligned}
$$

with probability one, in the sense that both limits exist together and are equal if they do. 
Similarly,

$$
\begin{aligned}
\lim _{n \rightarrow \infty} \frac{-1}{F_{\gamma}(n)} \sum_{j=1}^{n} \log \left(\frac{a_{\omega}(j)^{2}}{\tilde{a}(j)^{2}}\right) & =\lim _{n \rightarrow \infty} \frac{-1}{F_{\gamma}(n)} \sum_{j=1}^{n} \log \left(1+A_{\omega}(j)\right) \\
& =\lim _{n \rightarrow \infty} \frac{-1}{F_{\gamma}(n)} \sum_{j=1}^{n}\left(A_{\omega}(j)-\frac{A_{\omega}(j)^{2}}{2}\right) .
\end{aligned}
$$

Thus, our problem is reduced to computing the limits:

$$
\begin{gathered}
\xi_{\mathcal{Z}} \equiv \lim _{n \rightarrow \infty} \frac{1}{F_{\gamma}(n)} \sum_{j=1}^{n}\left(\left\|\mathcal{Z}_{\omega}(j)\right\|^{2}-1\right), \\
\xi_{\mathcal{W}} \equiv \lim _{n \rightarrow \infty} \frac{1}{F_{\gamma}(n)} \sum_{j=1}^{n}\left\|\mathcal{W}_{\omega}(j)\right\|^{2}, \\
\xi_{\mathcal{Z W}} \equiv \lim _{n \rightarrow \infty} \frac{2}{F_{\gamma}(n)} \sum_{j=1}^{n}\left(\mathcal{Z}_{\omega}(j), \mathcal{W}_{\omega}(j)\right), \\
\xi_{\mathcal{Z} \mathcal{W}_{2}} \equiv \lim _{n \rightarrow \infty} \frac{-2}{F_{\gamma}(n)} \sum_{j=1}^{n}\left(\mathcal{Z}_{\omega}(j), \mathcal{W}_{\omega}(j)\right)^{2}, \\
\xi_{A} \equiv \lim _{n \rightarrow \infty} \frac{-1}{F_{\gamma}(n)} \sum_{j=1}^{n}\left(A_{\omega}(j)-\frac{A_{\omega}(j)^{2}}{2}\right) .
\end{gathered}
$$

By (2.37),

$$
\begin{aligned}
\left\|\mathcal{Z}_{\omega}(j)\right\|^{2}-1= & \sin ^{2}\left(\bar{\theta}_{\omega}(j-1)\right) \frac{\tilde{a}(j)^{2}-\tilde{a}(j-1)^{2}}{\tilde{a}(j-1)^{2}} \\
& +\frac{\tilde{a}(j)^{2} \sin ^{2}\left(\bar{\theta}_{\omega}(j-1)\right)}{\tilde{a}(j-1)^{2}} \frac{\sin ^{2}\left(k_{j}\right)-\sin ^{2}\left(k_{j-1}\right)}{\sin ^{2}\left(k_{j-1}\right)}
\end{aligned}
$$

The last term on the right is absolutely summable $\left(=\mathcal{O}\left(n^{-1-2 \eta_{1}}\right)\right)$, so we only need to look at $\sin ^{2}\left(\bar{\theta}_{\omega}(j-1)\right) \frac{\tilde{a}(j)^{2}-\tilde{a}(j-1)^{2}}{\tilde{a}(j-1)^{2}}$. But, by $\sin ^{2}(\alpha)=\frac{1}{2}-\frac{\cos (2 \alpha)}{2}$ and by Proposition 2.5, we see that (recall $\left.\tilde{a}(j)=\lambda_{1} j^{-\eta_{1}}\right)$

$$
\begin{aligned}
\xi_{\mathcal{Z}} & =\frac{1}{2} \lim _{n \rightarrow \infty} \frac{1}{F_{\gamma}(n)} \sum_{j=1}^{n} \frac{\tilde{a}(j)^{2}-\tilde{a}(j-1)^{2}}{\tilde{a}(j-1)^{2}} \\
& =\frac{1}{2} \lim _{n \rightarrow \infty} \frac{1}{F_{\gamma}(n)} \sum_{j=1}^{n} \frac{2 \eta_{1}}{j}=\left\{\begin{array}{cc}
\eta_{1} & \text { if } \gamma \geq 1 / 2, \\
0 & \text { otherwise. }
\end{array}\right.
\end{aligned}
$$


For the other four limits, we shall use extensively Lemma 8.4 of [13] in order to replace $A_{\omega}$ and $B_{\omega}$ by their means. For example, write

$$
\begin{aligned}
\left\|\mathcal{W}_{\omega}(j)\right\|^{2}= & \frac{\sin ^{2}\left(\bar{\theta}_{\omega}(j-1)\right) \tilde{a}(j)^{2}}{\sin ^{2}\left(k_{j-1}\right) \tilde{a}(j-1)^{2}}\left(A_{\omega}(j)^{2}-\left\langle A_{\omega}(j)^{2}\right\rangle\right) \\
& +\frac{\sin ^{2}\left(\bar{\theta}_{\omega}(j-1)\right) \tilde{a}(j)^{2}}{\sin ^{2}\left(k_{j-1}\right) \tilde{a}(j-1)^{2}}\left(B_{\omega}(j)^{2}-\left\langle B_{\omega}(j)^{2}\right\rangle\right) \\
& +\frac{\sin ^{2}\left(\bar{\theta}_{\omega}(j-1)\right) \tilde{a}(j)^{2}}{\sin ^{2}\left(k_{j-1}\right) \tilde{a}(j-1)^{2}}\left(2 \cos \left(k_{j}\right) B_{\omega}(j) A_{\omega}(j)\right) \\
& +\frac{\sin ^{2}\left(\bar{\theta}_{\omega}(j-1)\right) \tilde{a}(j)^{2}}{\sin ^{2}\left(k_{j-1}\right) \tilde{a}(j-1)^{2}}\left(\left\langle A_{\omega}(j)^{2}\right\rangle+\left\langle B_{\omega}(j)^{2}\right\rangle\right) .
\end{aligned}
$$

Then the first three terms have mean zero, and so, by Lemma 8.4 of [13], we get that

$$
\xi_{\mathcal{W}} \equiv \lim _{n \rightarrow \infty} \frac{1}{F_{\gamma}(n)} \sum_{j=1}^{\infty} \frac{\sin ^{2}\left(\bar{\theta}_{\omega}(j-1)\right) \tilde{a}(j)^{2}}{\sin ^{2}\left(k_{j-1}\right) \tilde{a}(j-1)^{2}}\left(\left\langle A_{\omega}(j)^{2}\right\rangle+\left\langle B_{\omega}(j)^{2}\right\rangle\right)
$$

Expanding $\left\langle A_{\omega}(j)^{2}\right\rangle$, throwing out terms that are $o\left(j^{-2 \gamma}\right)$, and applying Proposition 2.5 (writing, again, $\sin ^{2}(\alpha)=\frac{1}{2}-\frac{\cos (2 \alpha)}{2}$ ), we get

$$
\begin{aligned}
\gamma_{\mathcal{W}}= & \lim _{n \rightarrow \infty} \frac{1}{F_{\gamma}(n)} \sum_{j=1}^{n} \frac{j^{2 \eta_{1}}}{(j-1)^{2 \eta_{1}} \sin ^{2}\left(k_{j-1}\right)} \Lambda j^{-2 \gamma} \\
& \times\left(\left\langle 4 Y_{\omega}(j)^{2}\right\rangle+\left\langle X_{\omega}(j)^{2}\right\rangle\right) \\
= & \lim _{n \rightarrow \infty} \frac{1}{F_{\gamma}(n)} \sum_{j=1}^{n} 2 \Lambda j^{-2 \gamma}=\left\{\begin{array}{cc}
0 & \text { if } \gamma>1 / 2 \\
2 \Lambda & \text { otherwise }
\end{array}\right.
\end{aligned}
$$

(recall $\left.\left\langle Y_{\omega}(j)^{2}\right\rangle=\frac{1}{4}\right)$. Applying the same procedure to $\xi_{\mathcal{Z W}}$ and $\xi_{A}$ we get

$$
\begin{aligned}
\xi_{\mathcal{Z W}} & =\lim _{n \rightarrow \infty} \frac{2}{F_{\gamma}(n)} \sum_{j=1}^{n} \frac{j^{2 \eta_{1}}}{(j-1)^{2 \eta_{1}}} \frac{\sin ^{2}\left(\bar{\theta}_{\omega}(j-1)\right)}{\sin ^{2}\left(k_{j-1}\right)}\left\langle 2 \Lambda \frac{Y_{\omega}(j)^{2}}{j^{2 \gamma}}\right\rangle \\
& =\lim _{n \rightarrow \infty} \frac{1}{F_{\gamma}(n)} \sum_{j=1}^{n} \frac{\Lambda}{2} \frac{1}{j^{2 \gamma}}=\left\{\begin{array}{cc}
0 & \text { if } \gamma>1 / 2, \\
\frac{\Lambda}{2} & \text { otherwise }
\end{array}\right.
\end{aligned}
$$

and

$$
\begin{aligned}
\xi_{A} & =\lim _{n \rightarrow \infty} \frac{-1}{F_{\gamma}(n)} \sum_{j=1}^{n}\left\langle 2 \Lambda \frac{Y_{\omega}(j)^{2}}{j^{2 \gamma}}\right\rangle \\
& =\lim _{n \rightarrow \infty} \frac{1}{F_{\gamma}(n)} \sum_{j=1}^{n}\left(\frac{\Lambda}{2} \frac{1}{j^{2 \gamma}}\right)= \begin{cases}0 & \text { if } \gamma>1 / 2 \\
\frac{\Lambda}{2} & \text { otherwise. }\end{cases}
\end{aligned}
$$


The computation of $\xi_{\mathcal{Z} \mathcal{W}_{2}}$ involves $\sin ^{4}\left(\bar{\theta}_{\omega}\right)=\frac{3}{8}-\frac{\cos \left(2 \bar{\theta}_{\omega}\right)}{2}+\frac{\cos \left(4 \bar{\theta}_{\omega}\right)}{8}$, for which Proposition 2.5 is useless. Luckily, the $\cos \left(4 \bar{\theta}_{\omega}(j)\right)$ cancels out. As before,

$$
\begin{aligned}
\xi_{\mathcal{Z} \mathcal{W}_{2}}= & \lim _{n \rightarrow \infty} \frac{-2}{F_{\gamma}(n)} \sum_{j=1}^{n} \frac{2 \Lambda}{j^{2 \gamma}} \\
& \times\left(\frac{\left\langle 4 Y_{\omega}(j)^{2}\right\rangle \sin ^{4}\left(\bar{\theta}_{\omega}(j-1)\right)}{\sin ^{2}\left(k_{j-1}\right)} \frac{j^{2 \eta_{1}}}{(j-1)^{2 \eta_{1}}}\right. \\
& \left.+\frac{\left\langle X_{\omega}(j)^{2}\right\rangle \sin ^{2}\left(2 \bar{\theta}_{\omega}(j-1)\right)}{4}\right) .
\end{aligned}
$$

Now write

$$
\begin{aligned}
( & \left.\frac{\left\langle 4 Y_{\omega}(j)^{2}\right\rangle \sin ^{4}\left(\bar{\theta}_{\omega}(j-1)\right)}{\sin ^{2}\left(k_{j-1}\right)} \frac{j^{2 \eta_{1}}}{(j-1)^{2 \eta_{1}}}+\frac{\left\langle X_{\omega}(j)^{2}\right\rangle \sin ^{2}\left(2 \bar{\theta}_{\omega}(j-1)\right)}{4}\right) \\
= & \left(\frac{\sin ^{4}\left(\bar{\theta}_{\omega}(j-1)\right)}{\sin ^{2}\left(k_{j-1}\right)} \frac{j^{2 \eta_{1}}}{(j-1)^{2 \eta_{1}}}+\frac{\sin ^{2}\left(2 \bar{\theta}_{\omega}(j-1)\right)}{4}\right) \\
= & \left(\frac{3 j^{2 \eta_{1}}}{8(j-1)^{2 \eta_{1}} \sin ^{2}\left(k_{j-1}\right)}+\frac{1}{8}\right) \\
& +\frac{j^{2 \eta_{1}}}{2(j-1)^{2 \eta_{1}} \sin ^{2}\left(k_{j-1}\right)} \cos \left(2 \bar{\theta}_{\omega}(j-1)\right) \\
& +\cos \left(4 \bar{\theta}_{\omega}(j-1)\right)\left(\frac{j^{2 \eta_{1}}}{(j-1)^{2 \eta_{1}} \sin ^{2}\left(k_{j-1}\right)}-1\right) \\
= & \left(\frac{3 j^{2 \eta_{1}}}{8(j-1)^{2 \eta_{1}} \sin ^{2}\left(k_{j-1}\right)}+\frac{1}{8}\right) \\
& +\frac{j^{2 \eta_{1}}}{2(j-1)^{2 \eta_{1}} \sin ^{2}\left(k_{j-1}\right)} \cos \left(2 \bar{\theta}_{\omega}(j-1)\right) \\
& +\cos \left(4 \bar{\theta}_{\omega}(j-1)\right)\left(\mathcal{O}\left(n^{-1}\right)+\mathcal{O}\left(n^{-2 \eta_{1}}\right)\right)
\end{aligned}
$$

to see that

$$
\xi_{\mathcal{Z} \mathcal{W}_{2}}=\left\{\begin{array}{cc}
0 & \text { if } \gamma>1 / 2 \\
-2 \Lambda & \text { otherwise }
\end{array}\right.
$$

where the $\cos \left(2 \bar{\theta}_{\omega}\right)$ term vanishes by Proposition 2.5 Summing up the various limits, the proposition is proved.

Proof of Theorem 2.2. By (2.15), the theorem follows from Proposition 2.3 ,

Theorem 1.1 follows almost immediately from Theorem 2.2 and Proposition 2.3 , As in the Schrödinger situation, the case $\gamma=\frac{1}{2}$ requires some subtle reasoning. 
We have established that, in this case, with probability one, equation (2.3) has a solution, $\psi$, with

$$
|\psi(n)|^{2} \asymp n^{\Lambda-\eta_{1}} .
$$

In order to use subordinacy theory, we need the existence of another solution with faster decay at infinity. The following is Lemma 8.7 of [13], formulated for general regular matrices:

Lemma 2.6. Let $u_{\phi}=(\cos \phi, \sin \phi) \in \mathbb{R}^{2}$. For any matrix $A \in G L_{2}(\mathbb{R})$ with $\operatorname{det}(A)=d>0$, let $\phi(A)$ be the unique $\phi \in\left(-\frac{\pi}{2}, \frac{\pi}{2}\right]$ with $\frac{1}{\sqrt{d}}\left\|A u_{\phi}\right\|=\sqrt{d}\|A\|^{-1}$. Define $\rho(A)=\frac{\left\|A u_{0}\right\|}{\left\|A u_{\pi / 2}\right\|}$.

Let $A_{n}$ be a sequence of matrices in $G L_{2}(\mathbb{R})$ with $\operatorname{det}\left(A_{n}\right)=d_{n}>0$ that satisfy

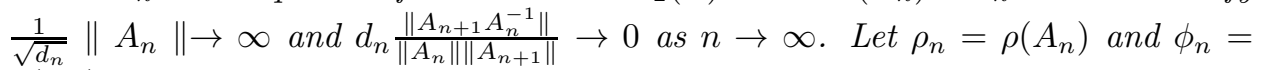
$\phi\left(A_{n}\right)$. Then:

(1) $\phi_{n}$ has a limit $\phi_{\infty}$ if and only if $\lim _{n \rightarrow \infty} \rho_{n}=\rho_{\infty}$ exists $\left(\rho_{\infty}=\infty\right.$ is allowed, but then we only have $\left.\left|\phi_{n}\right| \rightarrow \frac{\pi}{2}\right)$.

(2) Suppose $\phi_{n}$ has a limit $\phi_{\infty} \neq 0, \frac{\pi}{2}$ (equivalently, $\left.\rho_{\infty} \neq 0, \infty\right)$. Then

$$
\lim _{n \rightarrow \infty} \frac{\log \left\|A_{n} u_{\infty}\right\|-\log \sqrt{d_{n}}}{\log \left\|A_{n}\right\|-\log \sqrt{d_{n}}}=-1
$$

if and only if

$$
\limsup _{n \rightarrow \infty} \frac{\log \left|\rho_{n}-\rho_{\infty}\right|}{\log \left\|A_{n}\right\|-\log \sqrt{d_{n}}} \leq-2 .
$$

Proposition 2.7. Let $J_{\Upsilon, \omega}$ be the family of random Jacobi matrices described in Theorem 2.2 , with $\gamma=\frac{1}{2}$. Then, for any $E \in \mathbb{R}$, there exists, with probability one, an initial condition $\Psi_{\phi(\omega)}=\left(\begin{array}{c}\cos (\phi(\omega)) \\ \sin (\phi(\omega))\end{array}\right)$ such that

$$
\lim _{n \rightarrow \infty} \frac{\log \left\|T_{\omega}^{E}(n) \Psi_{\phi(\omega)}\right\|}{\log n}=-\frac{1}{2}\left(\Lambda+\eta_{1}\right)
$$

Proof. We imitate the proof of [13, Lemma 8.8]. By Proposition 2.3

$$
\lim _{n \rightarrow \infty} \frac{\log \left|R_{\omega, 0}(n)\right|}{\log n}=\frac{1}{2}\left(\Lambda+\eta_{1}\right)
$$

and

$$
\lim _{n \rightarrow \infty} \frac{\log \left|R_{\omega, \pi / 2}(n)\right|}{\log n}=\frac{1}{2}\left(\Lambda+\eta_{1}\right)
$$

for almost every $\omega$. By (2.12)- (2.13),

$$
R_{\omega, 0}(n) R_{\omega, \pi / 2}(n) \sin \left(\theta_{\omega, \pi / 2}(n)-\theta_{\omega, 0}(n)\right)=\tilde{a}(n) \sin k_{n}
$$

so that, for almost every $\omega$,

$$
\lim _{n \rightarrow \infty} \frac{\log \left|\theta_{\omega, 0}(n)-\theta_{\omega, \pi / 2}(n)\right|}{\log n}=-\Lambda
$$

$\left(\right.$ recall $\left.\tilde{a}(n)=\lambda_{1} n^{\eta_{1}}\right)$. 
Let $\rho_{\omega}(n)=\frac{R_{\omega, 0}(n)}{R_{\omega, \pi / 2}(n)}$. Then

$(2.51) L_{\omega}(n) \equiv \log \rho_{\omega}(n+1)-\log \rho_{\omega}(n)=\log \left(1+\mathcal{X}_{\omega, 0}(n)\right)-\log \left(1+\mathcal{X}_{\omega, \pi / 2}(n)\right)$,

where

$$
\mathcal{X}_{\omega, \phi}(n)=\left(\left\|\mathcal{Z}_{\omega, \phi}(n+1)+\mathcal{W}_{\omega, \phi}(n+1)\right\|^{2}-1\right) .
$$

Since $\mathcal{X}_{\omega, \phi}(j)=\mathcal{O}\left(\frac{1}{\sqrt{j}}\right)$ almost surely, we may apply a finite Taylor expansion to the above (using (2.36), (2.37) and (2.38) ) to see that, with probability one, for large enough $n$,

$$
\begin{aligned}
L_{\omega}(n)= & \Delta_{\omega}^{1}(n)\left(\sin ^{2}\left(\bar{\theta}_{\omega, 0}(n-1)\right)-\sin ^{2}\left(\bar{\theta}_{\omega, \pi / 2}(n-1)\right)\right) \\
& +\Delta_{\omega}^{2}(n)\left(\sin \left(2 \bar{\theta}_{\omega, 0}(n-1)\right)-\sin \left(2 \bar{\theta}_{\omega, \pi / 2}(n-1)\right)\right) \\
& +\Delta_{\omega}^{3}(n)\left(\sin \left(\bar{\theta}_{\omega, 0}(n-1)\right) \sin \left(\theta_{\omega, 0}(n-1)\right)\right. \\
& \left.-\sin \left(\bar{\theta}_{\omega, \pi / 2}(n-1)\right) \sin \left(\theta_{\omega, \pi / 2}(n-1)\right)\right) \\
& +\mathcal{O}\left(n^{-(1+\Lambda-\varepsilon)}\right)
\end{aligned}
$$

where

$$
\left\langle\Delta_{\omega}^{1}(n)\right\rangle=\left\langle\Delta_{\omega}^{2}(n)\right\rangle=\left\langle\Delta_{\omega}^{3}(n)\right\rangle=0
$$

and

$$
\left\langle\left(\Delta_{\omega}^{i}(n)\right)^{2}\right\rangle \leq \frac{C_{j}}{n}
$$

$i=1,2,3$. (The $\mathcal{O}\left(n^{-(1+\Lambda-\varepsilon)}\right)$ for the remainder follows from Lemma 2.1 and (2.50).)

A standard application of Kolmogorov's inequality and the Borel Cantelli Lemma shows that with probability one, for large enough $k$, and for each $i=1,2,3$,

$$
\sup _{m=2^{k-1}+1, \ldots, 2^{k}}\left|\sum_{j=2^{k-1}+1}^{m} \Delta_{\omega}^{i}(j)\right| \leq k
$$

and also

$$
\sup _{m=2^{k-1}+1, \ldots, 2^{k}-1}\left|\sum_{m}^{2^{k}} \Delta_{\omega}^{i}(j)\right| \leq k .
$$

Combining this with the fact that, with probability one, for large enough $n$,

$$
\begin{aligned}
& \left(\sin ^{2}\left(\bar{\theta}_{\omega, 0}(n-1)\right)-\sin ^{2}\left(\bar{\theta}_{\omega, \pi / 2}(n-1)\right)\right)=\mathcal{O}\left(n^{-\Lambda+\varepsilon}\right), \\
& \left(\sin \left(2 \bar{\theta}_{\omega, 0}(n-1)\right)-\sin \left(2 \bar{\theta}_{\omega, \pi / 2}(n-1)\right)\right)=\mathcal{O}\left(n^{-\Lambda+\varepsilon}\right)
\end{aligned}
$$

and

$$
\begin{aligned}
& \left(\sin \left(\bar{\theta}_{\omega, 0}(n-1)\right) \sin \left(\theta_{\omega, 0}(n-1)\right)\right. \\
& \left.\quad-\sin \left(\bar{\theta}_{\omega, \pi / 2}(n-1)\right) \sin \left(\theta_{\omega, \pi / 2}(n-1)\right)\right)=\mathcal{O}\left(n^{-\Lambda+\varepsilon}\right),
\end{aligned}
$$

it follows that

$$
\sum_{n=1}^{\infty} L_{\omega}(n)
$$


exists and

almost surely.

$$
\left|\sum_{n=N}^{\infty} L_{\omega}(n)\right| \leq C_{\omega} N^{-\Lambda+\varepsilon}
$$

Thus, with proability one, $\lim _{n \rightarrow \infty} \frac{R_{\omega, 0}(n)}{R_{\omega, \pi / 2}(n)}=\lim _{n \rightarrow \infty} \rho_{\omega}(n)=\rho_{\omega}(\infty)$ exists and

$$
\limsup _{n \rightarrow \infty} \frac{\log \left|\rho_{\omega}(n)-\rho_{\omega}(\infty)\right|}{\log n} \leq-\Lambda .
$$

Lemma 2.6 completes the proof (note that $d_{n} \equiv \operatorname{det} T_{\omega}^{E}(n)=\frac{1}{a_{\omega}(n)}$ ).

We are now ready to complete the

Proof of Theorem 1.1. (1) By Theorem 2.2 Fubini's Theorem, the fact that the distribution of $X_{\omega}(n)$ is absolutely continuous with respect to Lebesgue measure, and the theory of rank-one perturbations (20]), it follows that, with probability one, the spectral measure is supported on the set of energies where, for any $\varepsilon>0$ and sufficiently large $n,\left\|T_{\omega}^{E}(n)\right\|^{2} \leq n^{-\eta_{1}+\varepsilon}$. From Corollary 4.4 of 9 it now follows that the spectral measure is continuous with respect to $(1-\varepsilon)$-dimensional Hausdorff measure, for any $\varepsilon>0$. Thus the spectral measure is one-dimensional. Since $\psi(n)=$ $\left(\delta_{1},(J-z)^{-1} \delta_{n}\right)$ solves the eigenvalue equation for $z$ (away from $n=0$ ), Theorem A.1 and Wronskian conservation imply that if $z \in \mathbb{R}$ were to be outside of the spectrum, the transfer matrices would have to exhibit exponential growth. Since this is not the case, it follows that the spectrum is $\mathbb{R}$.

(2) In this case again, the fact that the spectrum is $\mathbb{R}$ follows from the polynomial bound on the transfer matrices in Theorem 2.2 and Theorem A.1 below. As for the properties of the spectral measure, these follow from Theorem 1.2 in 9 using (2.48), Proposition 2.7 and the theory of rank one perturbations.

(3) The existence, with probability one, of an exponentially decaying eigenfunction, for every $E \in \mathbb{R}$, follows from Theorem 2.2 and Theorem 8.3 of [16. Fubini and the theory of rank one perturbations imply that the spectral measure is supported, with probability one, on the set where these eigenfunctions exist. Comparing powers of $n$ in the exponent, Theorem A.1 implies that, as long as $\eta_{1}>2 \eta_{2}$, the spectrum fills $\mathbb{R}$.

\section{Appendix A. A Combes-Thomas estimate for Jacobi matrices WITH UNBOUNDED PARAMETERS}

This section presents a Combes-Thomas estimate, suitable for application to Jacobi matrices with unbounded off-diagonal terms. Also see [18 for a related result.

Theorem A.1. Let $J=J\left(\{a(n)\}_{n=1}^{\infty},\{b(n)\}_{n=1}^{\infty}\right)$ be a self-adjoint Jacobi matrix such that $0<a(n) \leq f(n)$ for a nondecreasing function $f(n)$. Let $z \in \mathbb{C}$ be such that $\operatorname{dist}(z, \operatorname{Spec}(J))=\sigma($ where $\operatorname{Spec}(J)$ is the spectrum of $J)$. Then

$$
\left|\left(\delta_{1},(J-z)^{-1} \delta_{N}\right)\right| \leq \frac{2 e}{\sigma} e^{-\alpha_{N} \cdot N},
$$


where $\alpha_{N}=\min \left(1, \frac{\sigma}{4 e f(N)}\right)$. In particular,

$$
\left|\left(\delta_{1},\left(J_{\Upsilon, \omega}-z\right)^{-1} \delta_{N}\right)\right| \leq \frac{2 e}{\sigma} e^{-C(\sigma, \omega) \cdot N^{1-\eta_{1}}}
$$

almost surely.

Remark. The monotonicity of $f$ is not essential. One may instead replace $f(N)$ in the formula for $\alpha_{N}$ by $\max (f(1), \ldots, f(N))$.

Proof. Let $R_{N}$ be the diagonal matrix defined by

$$
R_{N}(n, n)= \begin{cases}e^{\alpha_{N} \cdot n}, & n \leq N \\ e^{\alpha_{N} \cdot N}, & n>N\end{cases}
$$

Then

$$
e^{\alpha_{n} \cdot(N-1)}\left(\delta_{1},(J-z)^{-1} \delta_{N}\right)=\left(\delta_{1}, R_{N}^{-1}(J-z)^{-1} R_{N} \delta_{N}\right),
$$

so it suffices to bound $\left\|R_{N}^{-1}(J-z)^{-1} R_{N}\right\|$. Noting that $R_{N}^{-1}(J-z)^{-1} R_{N}=$ $\left(R_{N}^{-1}(J-z) R_{N}\right)^{-1} \equiv C_{N}(z)$, we may apply the resolvent identity to get

$$
C_{N}(z)=(J-z)^{-1}+C_{N}(z) \cdot\left(J-z-R_{N}^{-1}(J-z) R_{N}\right)(J-z)^{-1} .
$$

A simple computation shows that $\left\|J-z-R_{N}^{-1}(J-z) R_{N}\right\| \leq 2 e f(N) \alpha_{N}$ if $\alpha_{N} \leq 1$. Thus, by $\left\|(J-z)^{-1}\right\| \leq \frac{1}{\sigma}$, we see that

$$
\left\|C_{N}(z)\right\| \leq \frac{1}{\sigma}+C_{N}(z) \frac{2 e f(N)}{\sigma} \alpha_{N}
$$

so, by $\alpha_{N} \leq \frac{1}{2} \cdot \frac{\sigma}{2 e f(N)}$, we see that

$$
\left\|C_{N}(z)\right\| \leq \frac{2}{\sigma}
$$

which finishes the proof.

\section{ACKNOWLEDGMENTS}

We are grateful to Peter Forrester and Uzy Smilansky for presenting us with the problem that led to this paper. We also thank Yoram Last and Uzy Smilansky for many useful discussions. We thank the referee for helpful remarks.

This research was supported in part by THE ISRAEL SCIENCE FOUNDATION (grant no. 1169/06) and by grant no. 2002068 from the United States-Israel Binational Science Foundation (BSF), Jerusalem, Israel.

\section{REFERENCES}

[1] J. Berezanskii, Expansions in Eigenfunctions of Selfadjoint Operators, Transl. Math. Monographs, Vol. 17, Amer. Math. Soc., Providence, RI, 1968. MR0222718 (36:5768)

[2] J. Breuer, P. Forrester and U. Smilansky, Random discrete Schrödinger operators from random matrix theory, J. Phys. A: Math. Theor. 40 (2007), F1-F8. MR2307717 (2008c:82073)

[3] F. Delyon, Appearance of a purely singular continuous spectrum in a class of random Schrödinger operators, J. Stat. Phys. 40 (1985), 621-630. MR806718 (87j:81039)

[4] F. Delyon, B. Simon and B. Souillard, From power-localized to extended states in a class of one-dimensional disordered systems, Phys. Rev. Lett. 52 (1984), 2187-2189.

[5] F. Delyon, B. Simon and B. Souillard, From power pure point to continuous spectrum in disordered systems, Ann. Inst. H. Poincaré Phys. Théor. 42 (1985), 283-309. MR797277 (87d:35098)

[6] I. Dumitriu and A. Edelman, Matrix models for beta ensembles, J. Math. Phys. 43 (2002), 5830-5847. MR 1936554 (2004g:82044) 
[7] D. J. Gilbert and D. B. Pearson, On subordinacy and analysis of the spectrum of onedimensional Schrödinger operators, J. Math. Anal. Appl. 128 (1987), 30-56. MR915965 (89a:34033)

[8] J. Janas and S. Naboko, Spectral analysis of selfadjoint Jacobi matrices with periodically modulated entries, J. Funct. Anal. 191 (2002), 318-324. MR.1911189(2003d:47042)

[9] S. Jitomirskaya and Y. Last, Power-law subordinacy and singular spectra, I. Half-line operators, Acta Math. 183 (1999), 171-189. MR.1738043 (2001a:47033)

[10] R. Killip and I. Nenciu, Matrix models for circular ensembles, Int. Math. Res. Not., 50 (2004), 2665-2701. MR2127367 (2006h:82003)

[11] R. Killip and M. Stoiciu, Eigenvalue statistics for CMV matrices: From Poisson to clock via $C \beta E$, preprint math-ph/0608002.

[12] A. Kiselev and Y. Last, Solutions, spectrum and dynamics for Schrödinger operators on infinite domains, Duke Math. J. 102, (2000), 125-150. MR1741780(2001i:35224)

[13] A. Kiselev, Y. Last, and B. Simon, Modified Prüfer and EFGP transforms and the spectral analysis of one-dimensional Schrödinger operators, Commun. Math. Phys. 194 (1998), 1-45. MR.1628290 (99g:34167)

[14] S. Kotani and N. Ushiroya, One-dimensional Schrödinger operators with random decaying potentials, Commun. Math. Phys. 115 (1988), 247-266. MR931664 (89f:60069)

[15] Y. Last, Quantum dynamics and decompositions of singular continuous spectra, J. Funct. Anal. 142 (1996), 406-445. MR1423040 (97k:81044)

[16] Y. Last and B. Simon, Eigenfunctions, transfer matrices, and absolutely continuous spectrum of one-dimensional Schrödinger operators, Invent. Math. 135 (1999), 329-367. MR 1666767 (2000f:47060)

[17] M. Reed and B. Simon, Methods of Modern Mathematical Physics, I. Functional Analysis, Academic Press, New York, 1972. MR0493419 (58:12429a)

[18] J. Sahbani, Spectral properties of Jacobi matrices of certain birth and death processes, J. Operator Theory 56 (2006), 377-390. MR2282688(2008e:47082)

[19] B. Simon, Some Jacobi matrices with decaying potential and dense point spectrum, Commun. Math. Phys. 87 (1982), 253-258. MR684102 (85d:47033)

[20] B. Simon "Spectral analysis of rank one perturbations and applications". In Proc. Mathematical Quantum Theory, II. Schrödinger Operators, CRM Proceedings and Lecture Notes 8, edited by J. Feldman, R. Froese and L. Rosen, 109-149. American Mathematical Society, Providence, RI (1995). MR1332038 (97c:47008)

[21] B. Simon, Orthogonal Polynomials on the Unit Circle, vol. 2. American Mathematical Society Colloquium Publications, American Mathematical Society, Providence, Rhode Island, 2005. MR2105089(2006a:42002b)

Department of Mathematics 253-37, California institute of Technology, Pasadena, CALIFORNIA 91125

E-mail address: jbreuer@caltech.edu

Current address: Einstein Institute of Mathematics, The Hebrew University of Jerusalem, Jerusalem, 91904, Israel 